\title{
Microbubble trapping in inverted optical tweezers
}

Thomas J. Smart, Mehmet Burcin Unlu, Philip H. Jones

Thomas J. Smart, Mehmet Burcin Unlu, Philip H. Jones, "Microbubble trapping in inverted optical tweezers," Proc. SPIE 10347, Optical Trapping and Optical Micromanipulation XIV, 1034731 (25 August 2017); doi:

10.1117/12.2274033

SPIE Event: SPIE Nanoscience + Engineering, 2017, San Diego, California, United States 


\title{
Microbubble Trapping In Inverted Optical Tweezers
}

\author{
Thomas J. Smart ${ }^{*}, a$, Mehmet Burcin $\mathrm{Unlu}^{b}$, and Philip H. Jones ${ }^{a}$, \\ ${ }^{a}$ Department of Physics and Astronomy, University College London, Gower Street, London, \\ WC1E 6BT, UK; \\ ${ }^{b}$ Department of Physics, Bogazici University, Bebek/Istanbul, 34342, Turkey
}

\begin{abstract}
We have developed an inverted microscope optical tweezers for trapping and manipulation of microscopic gas bubbles. Trapping is achieved by a time-averaged optical trap using a rapidly-scanning Gaussian laser beam. Unlike holographic optical tweezers for microbubbles that employ a Laguerre-Gaussian beam, in this configuration the backwards-directed optical gradient force is sufficient to confine a microbubble against both the optical scattering force and the microbubble buoyancy. We have calibrated the optical trapping forces for microbubbles with a range of sizes, and determined the scanning trap configuration that produces the strongest confinement. Our system also includes a real-time "point-and-click" user interface for interactive selection, capture and isolation of individual microbubbles with optimal trap stiffness.
\end{abstract}

Keywords: optical tweezers, microbubbles

\section{INTRODUCTION}

Microbubble-enhanced ultrasound-induced sonoporation increases the permeability of cell membranes to bioactive materials. ${ }^{1}$ Since the cell membrane typically acts as an impenetrable barrier to water-soluble molecules, this enhanced uptake has clear implications for drug delivery and gene therapy. In order to better understand the role of microbubbles in sonoporation or to be able to develop efficient theranostic applications for microbubbles, it is crucial to have a means of manipulating and observing individual bubbles, both for studying the sonoporation process, and for evaluating and optimizing the properties of the bubbles themselves. ${ }^{2,3}$

The physical properties of gas microbubbles lend themselves to manipulation by either acoustic ${ }^{4}$ or optical $^{5}$ forces. Optical manipulation of microscopic gas bubbles has previously been demonstrated using either a timeaveraged (rapidly scanning) Gaussian laser beam, ${ }^{5}$ or an annular Laguerre-Gaussian beam. ${ }^{6}$ Both of these geometries have been realized using an upright microscope: the laser beam propagates downwards (antiparallel to gravity), thus the buoyancy of the microbubble is opposed to the scattering force from the laser beam. Indeed, in the case of the Laguerre-Gaussian beam, the microbubble is not optically trapped in three dimensions since the vertical equilibrium relies on the balance of scattering force and buoyancy, ${ }^{7}$ i.e. the inverse of 'optical levitation' for solid particles. ${ }^{8}$

Here we trap microbubbles using a scanning optical tweezers in an inverted configuration. In this geometry the direction of forces is as shown in figure 1(a), i.e. the microbubble buoyancy, $F_{\mathrm{B}}$, is parallel to the optical scattering force, $F_{\text {scat }}$, and antiparallel to the optical gradient force, $F_{\text {grad }}$. Clearly three-dimensional optical trapping will only be possible in this configuration if the backwards-directed gradient force is sufficient to balance the combination of the forward-directed scattering force and the microbubble buoyancy. Sung and Lee ${ }^{9}$ have calculated the net optical force on a microbubble in the scanning trap geometry, and found that strong and stable optical trapping is theoretically possible in both upright and inverted microscope configurations. In an experiment, however, the ability to trap the bubble will depend on factors that may be difficult to quantify for theoretical calculations, such as the amount of spherical aberration in the focused beam, and to our knowledge three-dimensional optical trapping in the inverted geometry has not previously been demonstrated.

\footnotetext{
* thomas.smart.13@ucl.ac.uk
} 


\section{EXPERIMENT SET-UP}

Our experimental configuration is shown in figure 1(b), and is the same as has been previously reported for experiments involving scanning or moving optical traps. ${ }^{10,11}$ In brief, the optical tweezers is built around a Zeiss Axiovert 200 inverted fluorescence microscope using a $\times 100$, NA $=1.3$ oil immersion objective lens for both trapping and imaging. The trapping laser beam is derived from a single-mode Nd:YAG laser with maximum output power of $3 \mathrm{~W}$, and beam steering is performed by a pair of galvanometer-scanning mirrors ${ }^{12}$ in a plane made conjugate to the back aperture of the objective by a pair of lenses, which also serve as a telescope to expand the beam such that it slightly overfills the objective back aperture. The microscope is also equipped with a three-axis nanopositioning stage. Control of the scanning mirrors and the piezo-stage, and thus threedimensional control of the microbubble within the sample chamber is performed via a custom-written National Instruments interface, and the experiment is visualized using a CMOS camera.

(a)

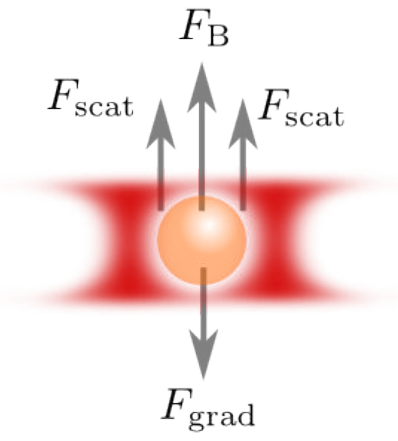

(b)
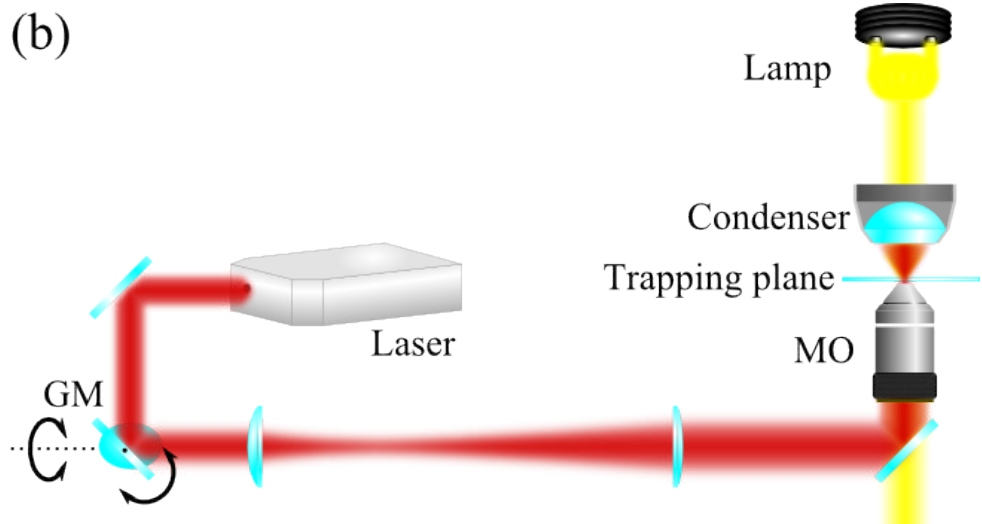

Lamp

CMOS Camera

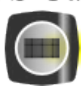

Figure 1. (a) Direction of forces on a microbubble in the inverted optical tweezers. In this geometry the buoyancy, $F_{\mathrm{B}}$, is parallel to the optical scattering force, $F_{\text {scat }}$, and antiparallel to the optical gradient force, $F_{\text {grad }}$. (b) Experimental set-up for optical trapping of microbubbles. The laser beam is directed via orthogonally-mounted steerable galvanometer mirrors (GM) and expanded in a telescope before being injected into the microscope objective (MO). The black arrows indicate the rotations of the galvanometer mirrors. The experiment is imaged using the CMOS camera, movie from which is used for digital video microscopy particle tracking and analysis.

The microbubbles used are Expancel (Akzo Nobel) which are expanded from their dry state using boiling water. $^{6}$ The sample chamber is made using a pain glass slide and a $120 \mu \mathrm{m}$ thick adhesive spacer (Invitrogen SecureSeal). The chamber is sealed using a No. 0 cover slip. The use of a thinner cover slip than the one for which the objective is designed is necessary since due to the microbubbles' buoyancy they reside at the top of the chamber, and the short working distance of the high NA objective precludes the use of a thicker (No. 1.5) cover slip. Both factors (the distance from the cover slip and the cover slip thickness) are likely to introduce spherical aberration in the beam which would reduce trapping efficiency, ${ }^{13}$ potentially inhibiting the ability to trap microbubbles in the inverted geometry.

Despite these limitations we are able to successfully trap microbubbles in three dimensions with this optical tweezers set-up and measure the stiffness of the optical trap in the transverse plane.

\section{RESULTS}

We first demonstrate that the inverted optical tweezers set-up is capable of optically trapping microbubbles. Figure 2 shows a sequence of images extracted from a video to demonstrate 3D trapping. In figure 2(a) two microbubbles can be seen resting against the upper surface of the trapping chamber as a result of their buoyancy. The bubble on the right is confined within a scanning optical tweezers with a scanning trap radius 1.3 times 
the bubble radius, i.e. $R_{\mathrm{T}} \simeq 1.3 \times r_{\mathrm{b}}$, and a frequency of $50 \mathrm{~Hz}$. In figure 2 (b) the microscope stage is raised a distance $\Delta z=16 \mu \mathrm{m}$ (i.e. approximately $4 r_{\mathrm{b}}$, which results in the images of both bubbles in the field of view becoming defocused. The defocussing of the bubbles on the right is, however, less as it is moved away from the trapping chamber surface in the axial direction by the optical trap. The bubble equilibrium axial position is determined by the balance of buoyancy and optical forces - as illustrated in figure 1(a) - and therefore even when trapped the bubble is held beyond the camera imaging plane. Manipulation of the trapped bubbles is demonstrated in figure $2(\mathrm{c})$ and $(\mathrm{d})$, where the trapped bubble is moved in the $x$ and $y$ directions respectively.
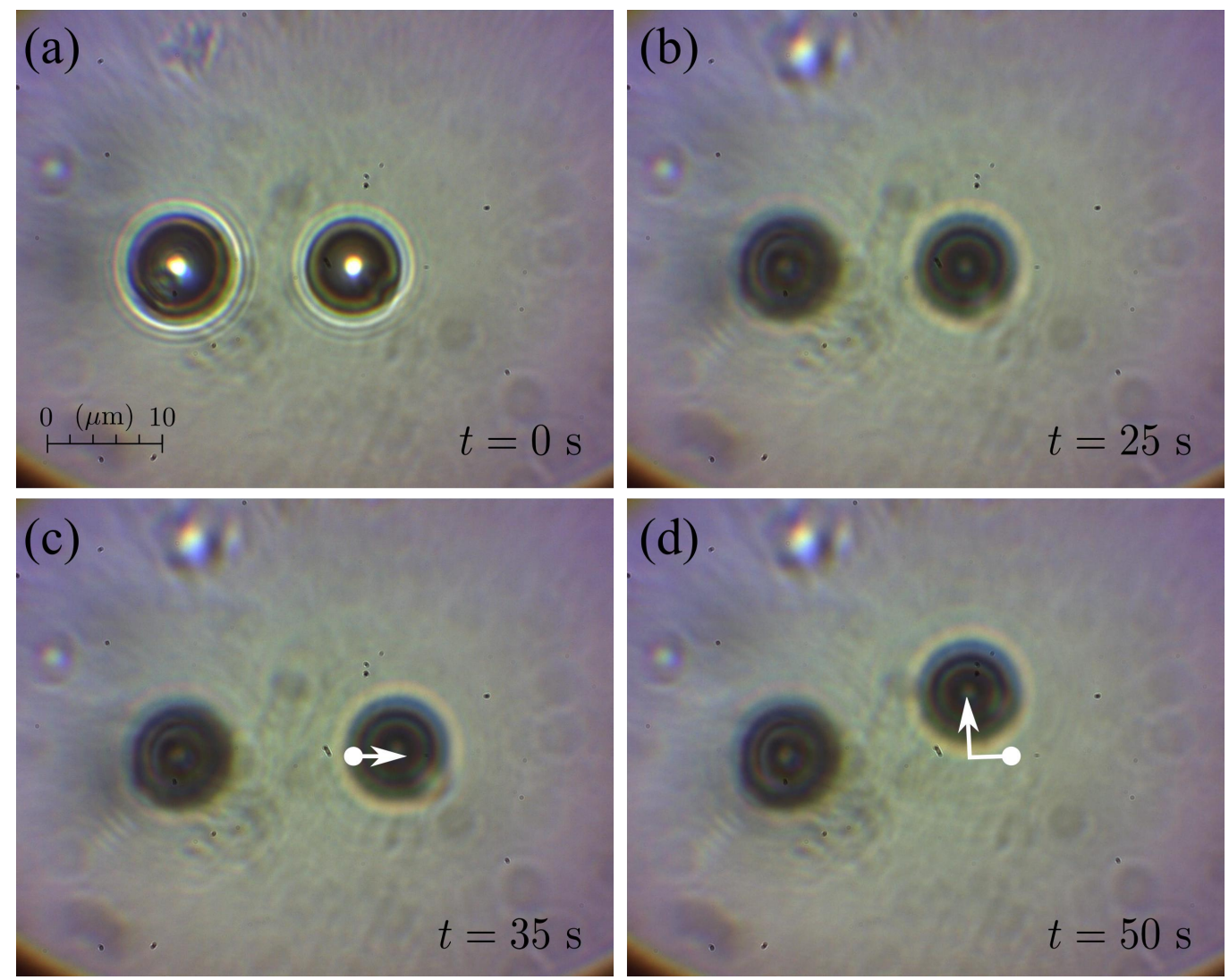

Figure 2. Demonstration of three-dimensional optical trapping of a microbubble in the inverted scanning optical tweezers. (a) Two microbubbles are resting against the upper surface of the trapping chamber, and the bubble on the right is confined within the trapping ring; (b) the microscope stage is raised to move the trapped bubble away from the surface. The combination of buoyancy and optical scattering forces lead to the bubble equilibrium position lying somewhat beyond the imaging plane of the camera; (c) transverse manipulation of the trapped bubble in the $x$ direction; (d) transverse manipulation of the bubble in the $y$ direction. In parts (c) and (d) the white arrows show the direction of bubble movement between frames.

To calibrate the optical trap for microbubbles we implement a video-based particle tracking system. ${ }^{13}$ Example tracking results are shown in figure 3(a), which shows a scatter plot of the centroid of the tracked, trapped microbubbles, clearly demonstrating the transverse confinement in the optical tweezers. The trajectories of the microbubble centroid in the $x$ and $y$ directions are shown in figures 3(b) and (c), and the histograms of these fluctuating co-ordinates in figures $3(\mathrm{~d})$ and (e). as expected for a overdamped, harmonically confined particle performing Brownian motion these distributions are (approximately) Gaussian, and we may extract the transverse trap stiffness by application of the theory of equipartition of energy. ${ }^{13}$

Example results of the trap stiffness measurement are shown in figure 4, which shown the average transverse stiffness, $\kappa_{\perp}=\left(\kappa_{x}+\kappa_{y}\right) / 2$ as a function of the trapping ring radius, $R_{\mathrm{T}}$, normalized by the bubble radius, $r_{\mathrm{b}}=3.6 \mu \mathrm{m}$ at fixed laser power. In these experiments we are able to investigate a wider range of trapping radius than previous work using an upright microscope set-up, ${ }^{12}$ and we find evidence of an optimum radius for 

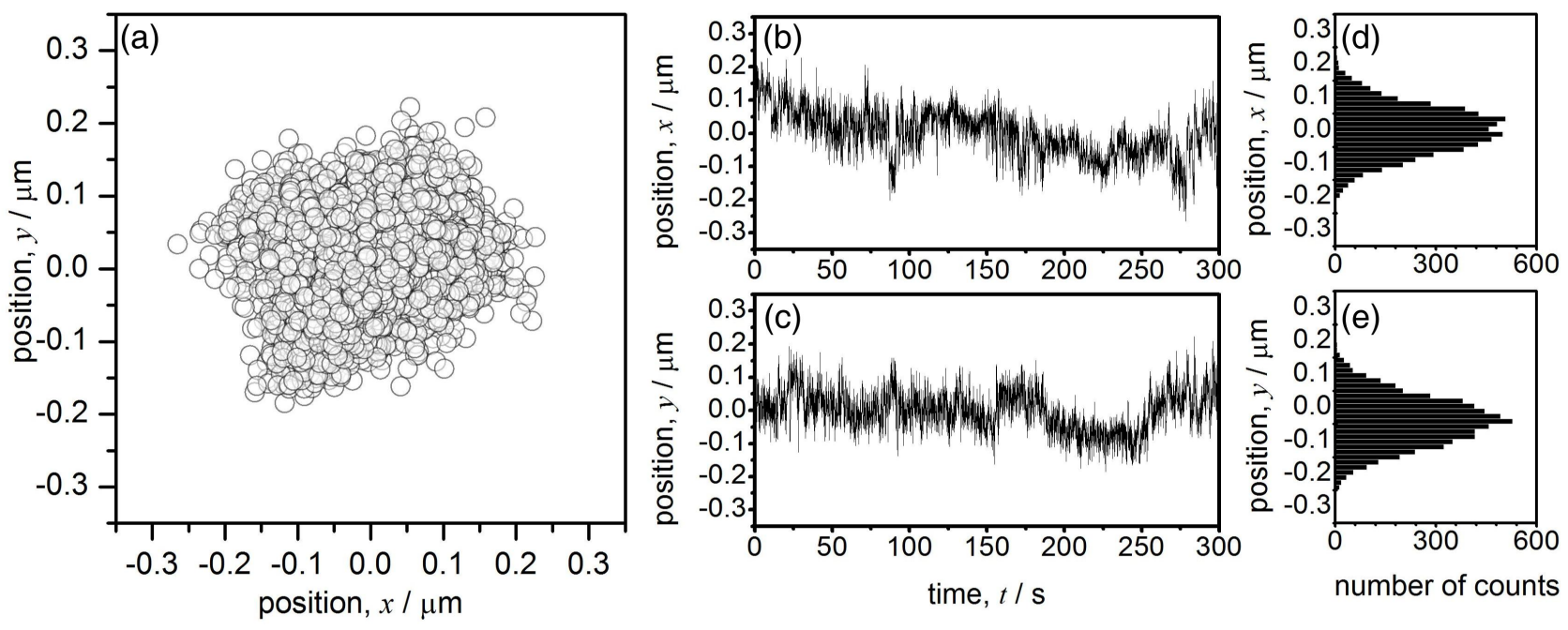

Figure 3. Resuts of video-based particle tracking of an optically trapped microbubble in the inverted scanning optical tweezer. (a) scatter plot of microbubble centroid motion in the transverse ( $x y$ ) plane; (b) trajectory of the centroid $x$ co-ordinate; (c) trajectory of the centroid $y$ co-ordinate; (d) histogram of the centroid $x$ co-ordinate; (e) histogram of the centroid $y$ co-ordinate.

the scanning trap at $R_{\mathrm{T}} \simeq 1.3 r_{\mathrm{b}}$ which maximizes the transverse stiffness. Further differences to the previous experiment include the numerical aperture of the microscope objective, and the wavelength of laser (and hence size of the focal spot). The existence of such a maximum can be qualitatively understood as a trade-off between a higher transverse intensity gradient, which would be achieved as smaller trap radii, and the higher scattering force that would occur which would push the bubble further from the waist to where transverse intensity gradients are lower.

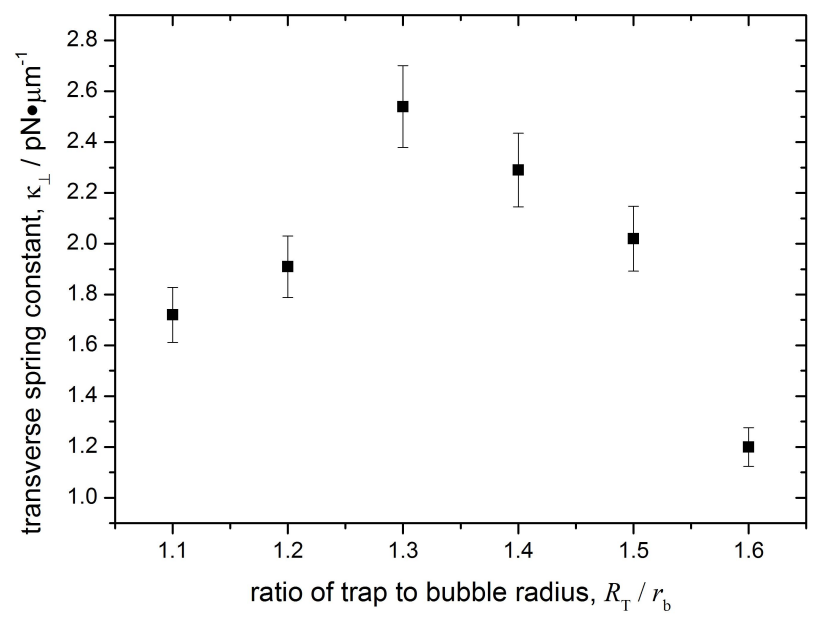

Figure 4. Measure transverse spring constant $\kappa_{\perp}=\left(\kappa_{x}+\kappa_{y}\right) / 2$ as a function of the trapping ring radius, $R_{\mathrm{T}} / r_{\mathrm{b}}$, showing the existence of a trap radius that maximizes $\kappa_{\perp}$.

Lastly, we have used the knowledge gained from calibration experiments to develop a real-time "point-andclick" user interface for trapping and manipulation of microbubbles, as shown in figure 5. Operation of the 
tweeers proceeds as follows. A microbubble is initially identified by the user and selected, shown in figure 5(a). Both the location and size of the selected microbubble are extracted from the video, and the galvanometer mirrors used to steer a scanning beam trap to the correct location with a diameter that optimizes the transverse trapping stiffness. The galvanometer mirrors are then used to steer the microbubble, either interactively by the user or to a pre-defined location, as illustrated in figure 5(b) - (d). The maximum speed of translation depends on the trap stiffness since displacement of the bubble from the trap ring center produces a greater overlap with the high intensity part of the scanning beam and thus a higher scattering force which pushes the bubble towards an escape trajectory. Optimizing the stiffness therefore increases the speed with which the bubble may be manipulated.
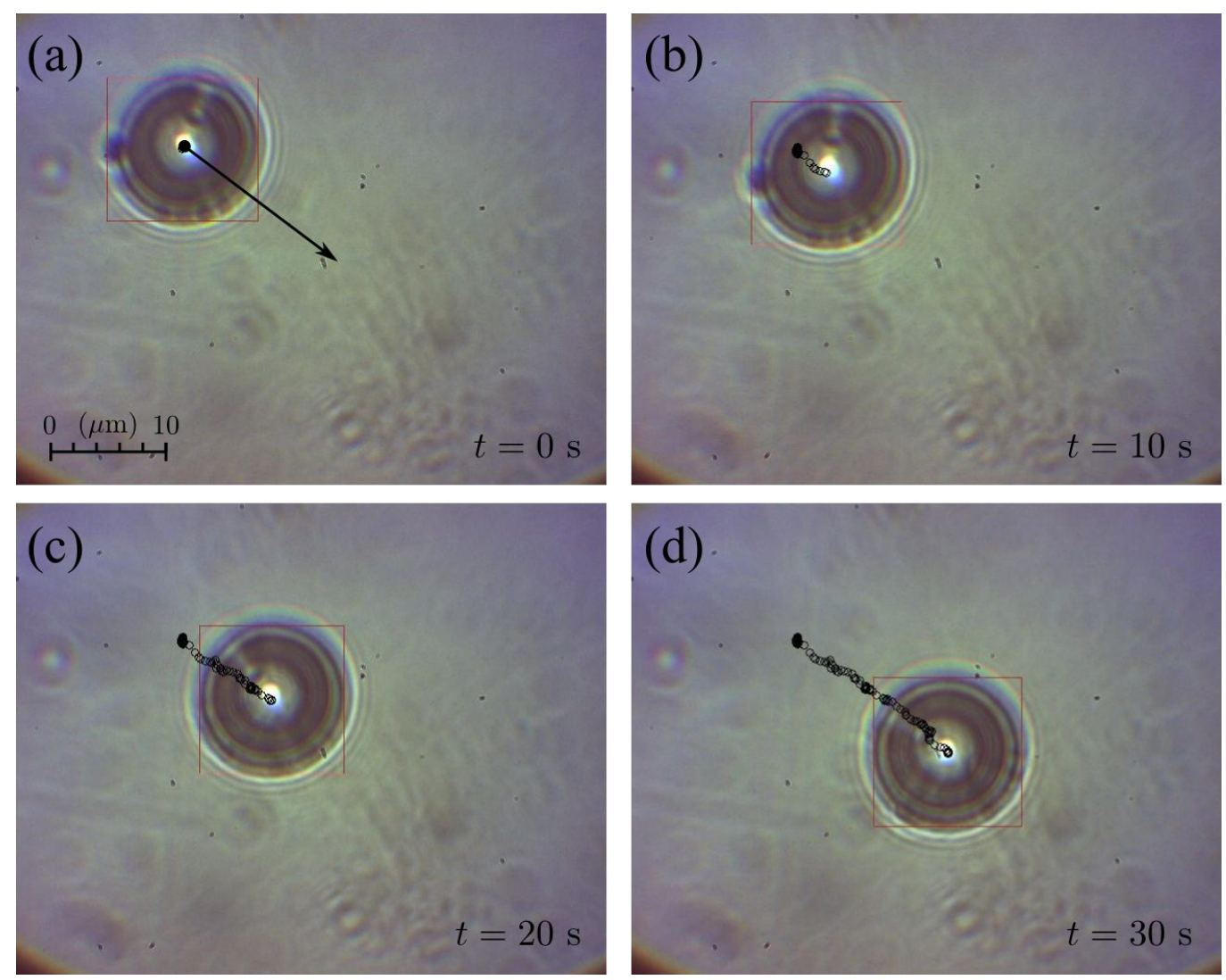

Figure 5. "Point-and-click" manipulation of an optically trapped microbubble. Frames taken from a movie at times (a) $t=0 \mathrm{~s}$; (b) $t=10 \mathrm{~s}$; (c) $t=20 \mathrm{~s}$; (d) $t=30 \mathrm{~s}$ from the start of the manipulation process. The black arrow shows the pre-defined direction of movement of the trap, and the black circles show the centroid of the bubble measured in the frames preceding those shown in the figure. The bubble has diameter $d=6.3 \pm 0.1 \mu \mathrm{m}$.

\section{CONCLUSION}

In conclusion, we have demonstrated an optical tweezers experiment for microbubbles suspended in water using an inverted microscope, that is capable of three-dimensional trapping using a scanning ring-shaped trap, even in the presence of strong buoyancy forces. We have calibrated the transverse stiffness of the optical trap using digital video microscopy, and found an optimum radius for the trapping ring. Using the calibration parameters we have demonstrated a user interface for facile selection and positioning of trapped microbubbles. We anticipate that such an instrument will be valuable for the study of microbubble dynamics in a controlled environment, and in particular their use in applications such as sonoporation and drug delivery.

\section{ACKNOWLEDGMENTS}

This work is supported by the British Council Newton Fund. 


\section{REFERENCES}

1. P. Prentice, A. Cuschieri, K. Dholakia, M. Prausnitz, and P. Campbell, "Membrane disruption by optically controlled microbubble cavitation," Nature Physics 1, pp. 107-110, 2005.

2. F. Saglimbeni, S. Bianchi, G. Bolognesi, G. Paradossi, and R. Di Leonardo, "Optical characterization of an individual polymer-shelled microbubble structure via digital holography," Soft Matter 8, pp. 8822-8825, 2012.

3. C. J. Harfield, C. R. Fury, G. Memoli, P. H. Jones, N. Ovenden, and E. P. J. Stride., "Analysis of the uncertainty in microbubble characterisation," Ultrasound in Medicine and Biology 42, pp. 1412-1418, 2016.

4. H. Erkol, E. Aytac-Kipergil, and M. B. Unlu, "Photoacoustic radiation force on a microbubble," Phys. Rev. E 90, p. 023001, 2014.

5. P. H. Jones, E. Stride, and N. Saffari, "Trapping and manipulation of microscopic bubbles with a scanning optical tweezer," Applied Physics Letters 89(8), p. 081113, 2006.

6. G. Memoli, C. R. Fury, K. O. Baxter, P. N. Gélat, and P. H. Jones, "Acoustic force measurements on polymer-coated microbubbles in a microfluidic device," The Journal of the Acoustical Society of America 141(5), pp. 3364-3378, 2017.

7. C. Fury, C. Harfield, P. H. Jones, E. Stride, and G. Memoli, "Experimental characterisation of holographic optical traps for microbubbles," Proc. SPIE 9126, p. 91263L, 2014.

8. A. Ashkin and J. M. Dziedzic, "Optical levitation by radiation pressure," Applied Physics Letters 19(8), pp. 283-285, 1971.

9. S.-Y. Sung and Y.-G. Lee, "Trapping of a micro-bubble by non-paraxial Gaussian beam: computation using the FDTD method," Opt. Express 16(5), pp. 3463-3473, 2008.

10. R. Agrawal, T. J. Smart, J. Nobre-Cardoso, C. J. Richards, R. Bhatnagar, A. Tufail, D. Shima, P. H. Jones, and C. Pavesio., "Assessment of red blood cell deformability in type 2 diabetes mellitus and diabetic retinopathy by dual optical tweezers stretching technique," Scientific Reports 6, p. 15873, 2016.

11. P. H. Jones, C. J. Richards, T. J. Smart, and D. Cubero, "Dynamical stabilisation in optical tweezers," Proc. SPIE 9379, p. 93790L, 2015.

12. P. H. Jones, O. M. Maragó, and E. P. J. Stride, "Parametrization of trapping forces on microbubbles in scanning optical tweezers," Journal of Optics A: Pure and Applied Optics 9(8), pp. S278-S283, 2007.

13. P. H. Jones, O. M. Maragó, and G. Volpe, Optical Tweezers: Principles $\&$ Applications, Cambridge University Press, Cambridge, first ed., 2015. 